\title{
Low sputum MMP-9/TIMP ratio is associated with airway narrowing in smokers with asthma
}

\author{
Rekha Chaudhuri', Charles McSharry', Jeffrey Brady², Christal Grierson², \\ C. Martina Messow ${ }^{5}$, Mark Spears ${ }^{1}$, Gino Miele ${ }^{2}$, Karl Nocka ${ }^{3}$, William MacNee ${ }^{6}$, \\ Martin Connell ${ }^{6}$, John T. Murchison ${ }^{6}$, Michael Sproule, Omar J. Hilmi ${ }^{8}$, \\ Douglas K. Miller ${ }^{4}$ and Neil C. Thomson ${ }^{1}$
}

\begin{abstract}
Affiliations: 'Institute of Infection, Immunity and Inflammation, University of Glasgow, Glasgow, UK. ${ }^{2}$ Pfizer Research/Translational Medicine Research Collaboration, Dundee, UK. ${ }^{3}$ Pfizer Research/Translational Medicine Research Collaboration, Cambridge MA, USA. "Pfizer Research/Translational Medicine Research Collaboration, Collegeville, PA, USA. ${ }^{5}$ Robertson Centre for Biostatistics, University of Glasgow, Glasgow, UK. ${ }^{6}$ University of Edinburgh/Medical Research Council Centre for Inflammation Research, Medical Physics and Clinical Radiology, University of Edinburgh, UK. ${ }^{7}$ Radiology Dept, Gartnavel General Hospital, Glasgow, UK. ${ }^{8}$ Ear Nose and Throat Dept, Gartnavel General Hospital, Glasgow, UK.
\end{abstract}

Correspondence: Rekha Chaudhuri, Institute of Infection, Immunity and Inflammation, University of Glasgow and Respiratory Medicine, Gartnavel General Hospital, Glasgow, G12 OYN, Scotland, UK. E-mail: rekhachaudhuridayahoo.com

ABSTRACT Asthmatic smokers have poor symptom control and accelerated decline in lung function. A reduced ratio of matrix metalloproteinase (MMP)-9/tissue inhibitors of metalloproteinases (TIMPs) in nonsmokers with asthma has been implicated in airway remodelling. We tested the hypothesis that sputum MMP-9 activity/TIMPs ratios are reduced in smokers compared with never-smokers with asthma and are associated with reduced lung function and altered computed tomography (CT) measures of airway wall dimensions.

Lung function, airway dimensions by CT, and induced sputum concentrations (and activity) of MMP-9 and TIMP-1 and -2 were measured in 81 asthmatics and 43 healthy subjects (smokers and never-smokers). Respiratory epithelial MMP9 and TIMP mRNA was quantified in 31 severe asthmatics and 32 healthy controls.

Sputum MMP-9 activity/TIMP-1 and TIMP-2 ratios, and nasal epithelial MMP9/TIMP1 and MMP9/ TIMP2 expression ratios were reduced in smokers with asthma compared with never-smokers with asthma. Low sputum ratios in asthmatic smokers were associated with reduced post-bronchodilator forced expiratory volume in $1 \mathrm{~s}$ (FEV1), FEV1/forced vital capacity ratio and segmental airway lumen area.

The association of a low sputum MMP-9 activity/TIMP-1 ratio with persistent airflow obstruction and reduced CT airway lumen area in smokers with asthma may indicate that an imbalance of MMP-9 and TIMPs contributes to structural changes to the airways in this group.

@ERSpublications

In asthmatic smokers, a low sputum MMP-9 activity/TIMP-1 is associated with spirometric and CT airway narrowing http://ow.ly/wnbAh

This article has supplementary material available from erj.ersjournals.com

Received: June 282013 | Accepted after revision: April 212014 | First published online: July 032014

Support statement: This work was funded by an award (INF-GU-090) from the Translational Medicine Research Collaboration, a consortium made up of the Universities of Glasgow, Edinburgh, Aberdeen and Dundee and the four associated NHS Health Boards (Greater Glasgow and Clyde, Lothian, Grampian, and Tayside), Scottish Enterprise and Pfizer (formerly Wyeth) and supported financially by NHS Research Scotland (NRS), through the Scottish Primary Care Research Network.

Conflict of interest: Disclosures can be found alongside the online version of this article at erj.ersjournals.com

Copyright @ERS 2014 


\section{Introduction}

A considerable proportion of patients with asthma, particularly those with severe disease [1], or who are cigarette smokers [2] have poorly controlled asthma, which can be associated with corticosteroid resistance [3], altered patterns of airway inflammation [4], airway remodelling [4] and persistent airflow obstruction [5]. The mechanisms leading to corticosteroid insensitivity and remodelling in asthma are poorly understood $[3,4]$.

Matrix metalloproteinases (MMPs) are zinc-dependent neutral endopeptidases that form a family of extracellular matrix (ECM) proteolytic enzymes. Activity of MMPs is regulated by specific protease inhibitors called tissue inhibitors of metalloproteinases (TIMPs). Inappropriate expression and activity of several MMPs, including MMP-9, and imbalances in the MMP-9/TIMP ratio have been implicated in the pathogenesis of asthma [6-12]. In nonsmokers with asthma a reduced sputum MMP-9/TIMP ratio is associated with more severe airway obstruction $[13,14]$ and with computed tomography $(\mathrm{CT})$ measures of increased airway wall area and thickness [14] as well as with increased CT lung scan abnormalities [15]. Based on these findings it has been proposed that high concentrations of TIMP-1 in relation to MMP-9 can lead to increased ECM deposition and increased myofibroblast proliferation, through TIMP-1 induced cell growth, and that a low sputum MMP-9/TIMP ratio may be a biomarker of airway remodelling in asthma [10-12]. Studies of asthma have recruited predominantly never-smokers; much less is known about MMP-9 activity and TIMP in smokers with asthma. Acute exposure of bronchial epithelial cells from patients with asthma to cigarette smoke in vitro reduces the MMP-9/TIMP-1 ratio suggesting that an interaction between asthma and cigarette smoking may potentiate structural changes to the airways [16]. We tested the hypothesis that MMP-9 activity/TIMP ratios are reduced in sputum from smokers with asthma compared with never-smokers with asthma and are associated with reduced lung function and altered CT measures of airway wall dimensions.

\section{Materials and methods \\ Subjects}

Participants were recruited with mild, moderate and severe persistent asthma (Global Initiative for Asthma classification) [17]; participants with asthma included both current smokers and never-smokers, and healthy smokers and never-smokers. All subjects were on stable medication and had had no exacerbation of disease for 4 weeks. Smokers were defined as individuals with $\geqslant 10$ pack-years who currently smoke five or more cigarettes per day. Asthma subjects had to demonstrate either reversibility of forced expiratory volume in $1 \mathrm{~s}$ (FEV1) or airway hyperresponsiveness. The West Glasgow Research Ethics Committee approved the study and all patients gave written informed consent.

\section{Study design}

The study was designed to examine the relationship between sputum MMP-9 activity/TIMP-1 and TIMP-2 ratios and the expression of MMP-9/TIMP-1 and MMP-9/TIMP-2 with sputum cytology, lung function and CT measures of airway dimensions in smokers and never-smokers with asthma. It was a cross-sectional study, performed in subjects with asthma and healthy controls, as part of a chronic obstructive pulmonary disease and asthma biomarker study [18].

\section{Measurements}

Additional details of the measurements can be found in the online supplementary material.

Spirometry was performed according to American Thoracic Society guidelines [19] and airway hyperresponsiveness to methacholine was measured. Reversibility was defined as $>12 \%$ and $200 \mathrm{~mL}$ improvement in FEV1 following salbutamol.

Exhaled nitric oxide fraction was measured (Niox Flex; Aerocrine, Solna, Sweden) at $50 \mathrm{~mL} \cdot \mathrm{s}^{-1}\left(\mathrm{FeNO}_{50}\right)$ in concordance with standardised guidelines [20].

Lung volumes and diffusing capacity of the lung for carbon monoxide (DLCO) were performed using the body box technique (Zan 500 Body Plethysmography; nSpire Health Limited, Hertford, UK).

Sputum induction was performed as previously described [18].

CT scans of the chest were performed at full inspiration using 16-slice Brightspeed and 64-slice Lightspeed (GE Healthcare, Milwaukee, WI, USA) with the following parameters: $120 \mathrm{KV}, 100 \mathrm{~mA} \cdot \mathrm{s}$, collimation $1 \mathrm{~mm}$, reconstruction slice thickness $0.65 \mathrm{~mm}$, reconstruction slice separation $0.5 \mathrm{~mm}$ and pitch of one. The data were reconstructed with a CHEST filter. All scans were evaluated centrally at the University of Edinburgh (Edinburgh, UK). Airway dimensions were measured using the software Pulmonary Workstation 2.0 (VIDA Diagnostics, Iowa City, IA, USA) to plot an airway path from which airway 
profiles were generated on cross-sections orthogonal to the airway path. Airway dimensions were measured in the right lower lobe posterior basal segmental bronchus (designated RB10). The following CT airway values were obtained: RB10 \% wall area; RB10 wall thickness $(\mathrm{mm})$; RB10 lumen area $\left(\mathrm{mm}^{2}\right)$.

MMP-9 assays were carried out using the MMP-9 enzyme immunoassay and MMP-9 activity kits (catalogue numbers DMP900 and F9M00, respectively; R\&D Systems Europe Ltd, Abingdon, UK). TIMPs were quantified by using fluorokine multiplex assay (LKT003; R\&D Systems Europe Ltd).

MMP-9 (Affymetrix number 203936_s_at), TIMP-1 (201666_at) and TIMP-2 (231579_s_at) mRNA expression levels were analysed using Affymetrix U133+2 chips (Affymetrix, Santa Clara, CA, USA) and quantified by global normalisation to sputum cell and nasal respiratory epithelial cell samples obtained from healthy never-smokers $(n=15)$, healthy smokers $(n=13)$, smokers with asthma $(n=15)$ and never-smokers with asthma $(n=15)$. Samples were selected by asthma severity and quality of RNA. Details of RNA isolation and quality are included in the online supplementary material.

\section{Analysis}

Continuous variables were summarised as median (interquartile range). Their comparison between different patient groups was by Wilcoxon test or Kruskal-Wallis test. Categorical variables were summarised as frequencies and percentages per category and were compared using Fisher's exact test. Linear regression models were used to predict each marker from disease group, smoking status and additional covariates, testing for any group by smoking status interaction. Associations of log-transformed MMP-9/TIMP ratios with sputum neutrophil percentage and CT RB10 airway dimension measurements were assessed using the Pearson correlation coefficient with bootstrap 95\% confidence intervals. The analyses were exploratory for the purpose of hypothesis generation, and statistical significance was accepted if $\mathrm{p}<0.05$. All analyses were carried out in R version 2.15.0 (The R Project for Statistical Computing. www.r-project.org).

\section{Results}

Demographics and baseline characteristics

81 asthmatics (35 smokers and 46 never-smokers) and 43 healthy subjects (19 smokers and 24 neversmokers) took part in the study. The asthma and healthy control groups were matched for age and smoking histories (table 1). None of the patients were on oral corticosteroids or anti-interleukin-5 therapy. The never-smokers with asthma were similar to the smokers with asthma in terms of age, duration of asthma, body surface area, atopic status, spirometry, dose of inhaled corticosteroid, treatment with montelukast, induced sputum total cell counts, eosinophil and neutrophil proportion, and CT measurements of airway wall thickness, but had a higher DLCO $(86 \%$ versus $77 \%$ predicted, $\mathrm{p}=0.002)$ and $\mathrm{FeNO}_{50}(23 \mathrm{ppb}$ versus $9 \mathrm{ppb}, \mathrm{p}<0.001)$.

\section{Sputum MMP-9/TIMP activity and concentration ratios}

The ratio of sputum MMP-9 activity to sputum TIMP-1 or sputum TIMP-2 was lower in smokers with asthma compared with never-smokers with asthma $(\mathrm{p}=0.002$ and $\mathrm{p}=0.001$ respectively), and in healthy smokers compared with healthy never-smokers $(p=0.018$ and $p=0.024$, respectively) (fig. 1 and table 2). The difference in these ratios was due to elevated sputum TIMP concentrations in smokers compared to never-smokers (in both asthma and healthy controls) (table 2). There was no significant difference between groups according to asthma severity (table 3 ).

The ratios of sputum MMP-9 concentration to sputum TIMP-1 or sputum TIMP-2 were similar between smokers with asthma and never-smokers with asthma and between both asthma groups and healthy neversmokers and smokers, respectively (fig. 1 and table 2). There was no significant difference between groups according to asthma severity (table 3 ).

Linear regression analyses of interaction demonstrated that neither age nor dose of inhaled corticosteroid had a significant effect on MMP-9/TIMP ratios and that smoking had a significant effect on MMP-9 activity/ TIMP-1 $(\mathrm{r}=-0.738(95 \% \mathrm{CI}-1.276--0.200), \mathrm{p}=0.008)$ and on MMP-9 activity/TIMP-2 ratios $(\mathrm{r}=-0.518$ (95\% CI -0.960--0.077), $\mathrm{p}=0.022$ ).

\section{Nasal respiratory epithelial cell and sputum cell MMP-9/TIMP expression ratios}

Median (interquartile range) nasal respiratory epithelial cell MMP9/TIMP1 and MMP9/TIMP2 expression ratios were lower in smokers with severe asthma compared with never-smokers with severe asthma $(0.01$ $(0.00-0.03)$ versus $0.05(0.04-0.09) \times 10^{-3}$ relative fluorescence units $(\mathrm{RFU}), \mathrm{p}<0.001$ and $0.02(0.01-0.06)$ versus $0.10(0.05-0.14) \times 10^{-3} \mathrm{RFU}, \mathrm{p}<0.001$, respectively). Ratios were also lower in healthy smokers compared with healthy never-smokers $(\mathrm{p}<0.001$ for both ratios) (online supplementary table E1). Sputum cell MMP9/TIMP1 and TIMP2 expression ratios were higher in smokers with severe asthma compared with 
TABLE 1 Demographics and clinical baseline characteristics

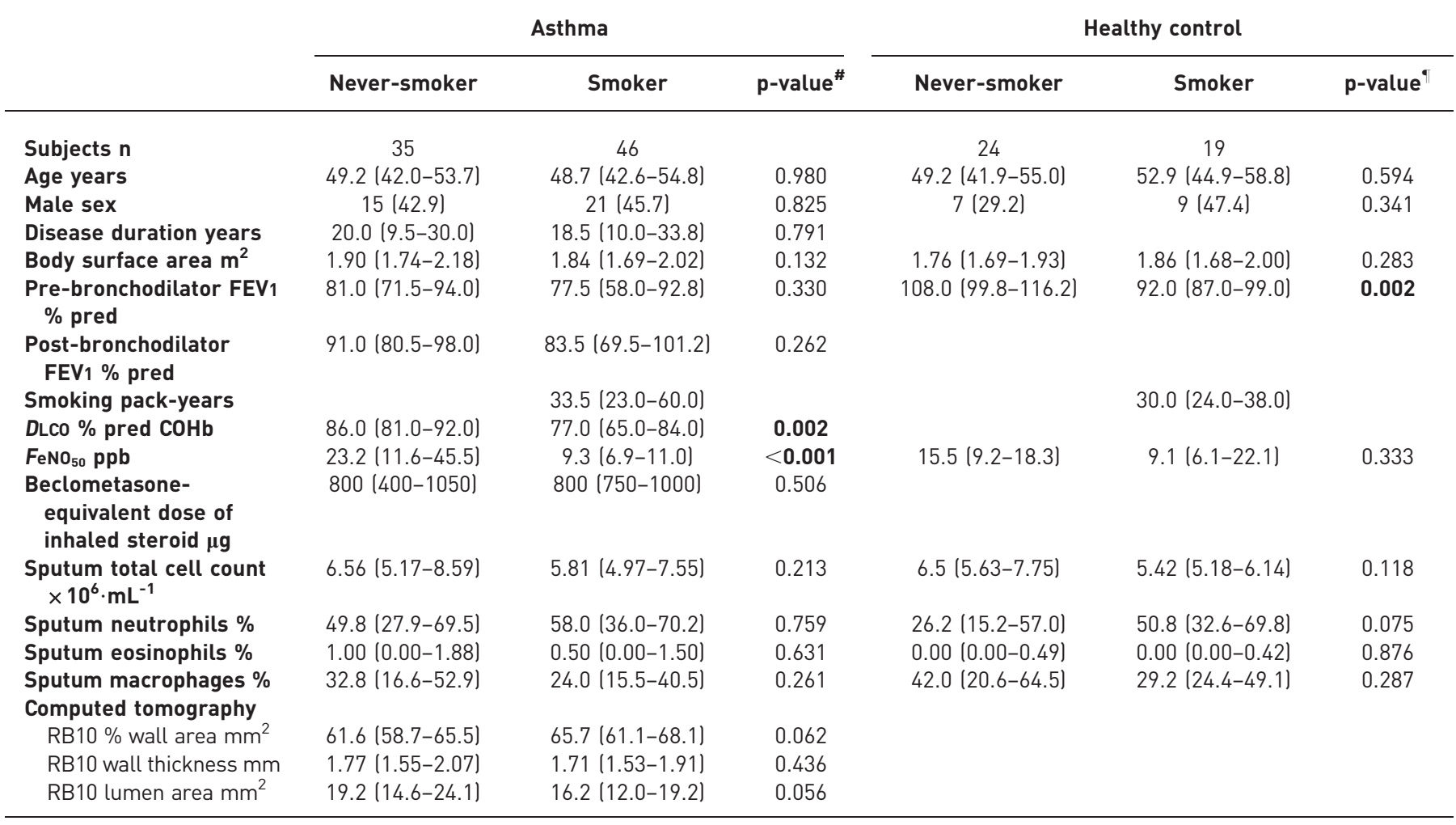

Data are presented as median (interquartile range) or $n(\%)$, unless otherwise stated. Bold font indicates $p<0.05$. FEV 1 : forced expiratory volume in $1 \mathrm{~s} ; \mathrm{LLCO} \%$ pred $\mathrm{COHb}$ : diffusing capacity of the lung for carbon monoxide corrected for haemoglobin and carboxyhaemoglobin, as a percentage of the predicted value; $\mathrm{FeNO}_{50}$ : exhaled nitric oxide fraction measured at a flow rate of $50 \mathrm{~mL} \cdot \mathrm{s}^{-1}$; RB10: right bronchial division $10 .{ }^{\#}$ : comparison of never-smokers with asthma and smokers with asthma; ${ }^{\circ}$ : comparison of healthy never-smokers and healthy smokers.

never-smokers with severe asthma $(0.19(0.09-0.27)$ versus $0.13(0.06-0.18), \mathrm{p}<0.001$ and $0.27(0.12-0.38)$ versus 0.13 (0.05-0.18), $\mathrm{p}=0.046$, respectively) (online supplementary table E1).

Correlation of sputum MMP-9 protein and enzyme activity and TIMP concentrations with lung function, sputum neutrophil percentage and CT scan measures of airway dimensions Lung function

In smokers with asthma sputum MMP-9 protein activity/TIMP-1 and TIMP-2 ratios were positively associated with post-bronchodilator FEV1 \% pred ( $\mathrm{r}=0.359$ (95\% CI 0.065-0.599) and 0.334 (0.008-0.587), respectively) and FEV1/forced vital capacity (FVC) ratio $(0.365(0.070-0.626)$ and $0.386(0.105-0.614)$, respectively) (table 4). There were no significant associations with DLCO, except for sputum MMP-9/TIMP-2 ratio in never-smokers with asthma $(-0.344(-0.621--0.028))$.

\section{Sputum neutrophil percentage}

In never-smokers with asthma, MMP-9 activity/TIMP-1 and MMP-9 concentration/TIMP-1 were positively associated with sputum neutrophil percentage $(0.373$ (95\% CI $0.020-0.697)$ and 0.579 (0.251-0.797), respectively) (table 4). Associations of MMP-9 concentration and activity/TIMP-2 were not significant in never-smokers with asthma, nor were any associations with sputum neutrophil percentage significant in smokers with asthma.

\section{CT measure of airway wall dimensions}

Sputum MMP-9 activity/TIMP-1 and TIMP-2 and sputum MMP-9 protein concentration/TIMP-1 and TIMP-2 ratios were positively associated with RB10 airway lumen area in smokers with asthma $(\mathrm{r}=0.450$ (95\% CI 0.138-0.717), 0.410 (0.102-0.668), 0.499 (0.189-0.728) and 0.320 (0.000-0.579), respectively) (fig. 2 and table 4). Sputum MMP-9/TIMP ratios were not significantly associated with RB10 airway wall thickening. There were no significant associations between the ratios and RB10 CT measures in 

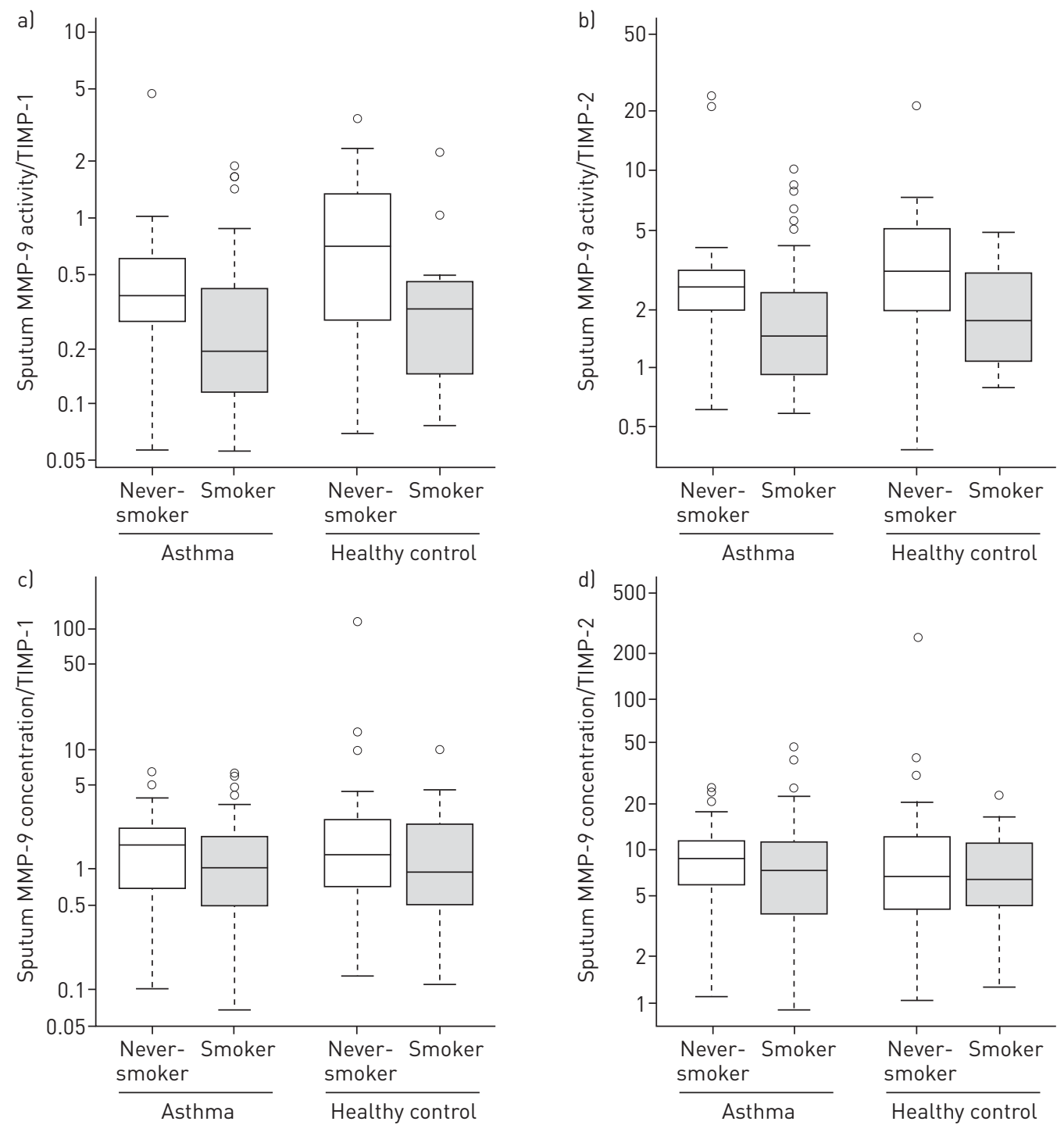

FIGURE 1 a) Sputum matrix metalloproteinase (MMP)-9 activity/tissue inhibitor of metalloproteinase (TIMP)-1 concentration ratio, b) MMP-9 activity/TIMP-2 concentration ratio, c) MMP-9 protein concentration/TIMP-1 concentration ratio, and d) MMP-9 protein concentration/TIMP-2 concentration ratio in patients with asthma and healthy controls. The ratio of sputum MMP-9 activity with sputum TIMP-1 concentration or sputum TIMP-2 concentration was lower in smokers with asthma compared with never-smokers with asthma $(\mathrm{p}=0.002$ and $\mathrm{p}=0.001$, respectively) and in healthy smokers compared with healthy never-smokers ( $p=0.018$ and $p=0.024$, respectively).

never-smokers with asthma. RB10 airway lumen area in smokers with asthma and never-smokers with asthma did not correlate with body surface area $(r=0.208, p=0.170$ and $r=0.066, p=0.680$, respectively).

\section{Discussion}

A reduced ratio of MMP-9/TIMPs in nonsmokers with asthma has been implicated in airway remodelling $[8,10-12]$. We sought to determine if the ratio of sputum MMP-9/TIMPs is associated with lung function or CT measures of airway dimensions in smokers and never-smokers with asthma. Particular strengths of our study were recruitment of smokers with asthma, who account for up to a third of all adults with asthma, and yet in whom there are limited published data describing the balance between MMP-9 and TIMPs, or CT imaging to assess airway wall dimensions. We also describe the use of functional assays for MMP-9 activity, which adds information about the proportion of the enzyme that becomes activated.

Our main new findings were that sputum MMP-9 activity/TIMP ratios were reduced in smokers with asthma compared with never-smokers with asthma and that reduced ratios in smokers with asthma were associated with reduced post-bronchodilator FEV1/FVC ratio and reduced airway lumen area as measured 


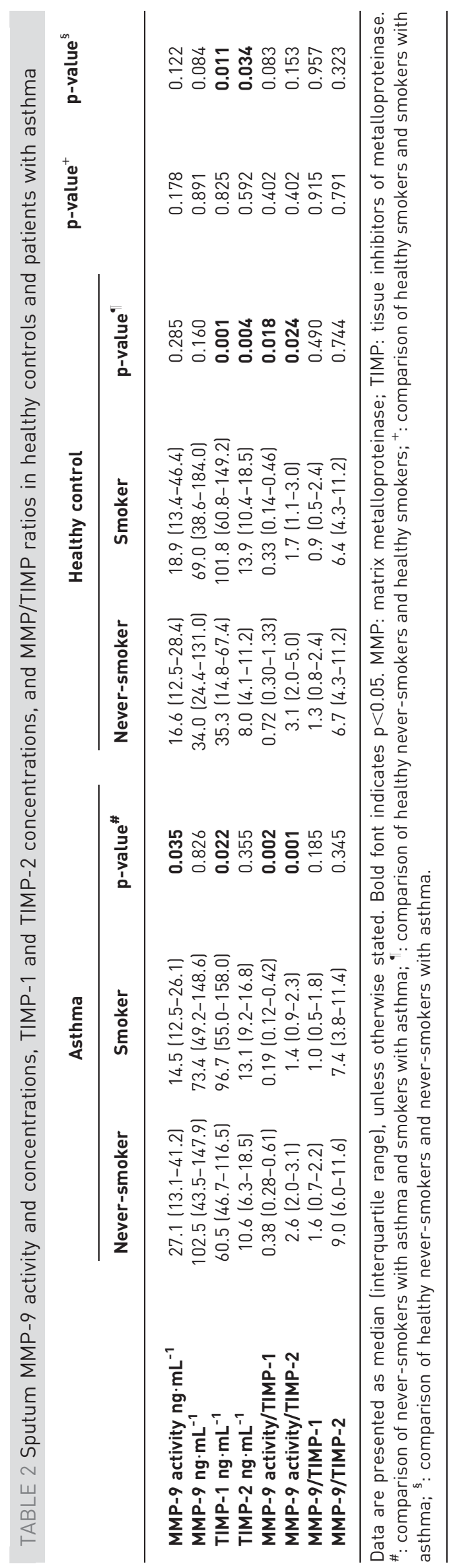


TABLE 3 Sputum MMP-9 activity and concentrations, TIMP-1 and TIMP-2 concentrations, and MMP/TIMP ratios and induced sputum cell cytology in patients with asthma, subdivided by severity and smoking

\begin{tabular}{|c|c|c|c|c|c|c|}
\hline & \multicolumn{3}{|c|}{ Never-smokers with asthma } & \multicolumn{3}{|c|}{ Smokers with asthma } \\
\hline $\begin{array}{l}\text { MMP-9 activity } \\
\mathrm{ng} \cdot \mathrm{mL}^{-1}\end{array}$ & $29.4(17.5-40.5)$ & $21.8(16.5-32.0)$ & $31.6(12.5-55.8)$ & $18.9(13.0-47.4)$ & $14.3(12.5-44.0)$ & $13.5(12.5-23.2)$ \\
\hline MMP-9 $\mathrm{ng} \cdot \mathrm{mL}^{-1}$ & $106(69-156)$ & $90(40-143)$ & $97(42-140)$ & $67(48-144)$ & $71(38-355)$ & $80(55-123)$ \\
\hline $\begin{array}{l}\text { MMP-9 activity/ } \\
\text { TIMP-1 }\end{array}$ & $0.4(0.4-0.6)$ & $0.3(0.2-0.5)$ & $0.4(0.2-0.8)$ & $0.2(0.1-0.7)$ & $0.3(0.2-0.4)$ & $0.1(0.1-0.3)$ \\
\hline $\begin{array}{l}\text { MMP-9 activity/ } \\
\text { TIMP-2 }\end{array}$ & $2.9(2.4-3.1)$ & $2.0(1.8-2.6)$ & $2.5(1.5-3.5)$ & $1.4(1.2-3.8)$ & $1.9(1.3-2.7)$ & $1.3(0.9-1.6)$ \\
\hline MMP-9/TIMP-1 & $1.7(1.1-2.2)$ & $1.6(0.7-2.1)$ & $1.3(0.6-1.8)$ & $1.3(0.4-2.0)$ & $1.2(0.5-1.4)$ & $0.9(0.6-1.7)$ \\
\hline MMP-9/TIMP-2 & $10.2(9.2-13.0)$ & $8.5(6.0-11.8)$ & $7.4(4.3-9.3)$ & $7.3(3.5-11.2)$ & $6.8(3.8-13.6)$ & $7.4(4.6-10.0)$ \\
\hline
\end{tabular}

Data are presented as median (interquartile range), unless otherwise stated. MMP: matrix metalloproteinase; TIMP: tissue inhibitors of metalloproteinase.

using CT. These findings suggest that airway narrowing is affected by the functional balance between MMP-9 and its inhibitors, which appears crucial in the dynamic process of airway remodelling and collagen deposition in asthma $[8,9,21]$. We also found reduced ratios of MMP9/TIMP1 and MMP9/TIMP2 mRNA expression levels in nasal respiratory epithelium in the subset of smokers with severe asthma, suggesting that these changes occurred in the sub-epithelial compartment where MMPs have to be activated and their interactions with TIMPs are locally regulated [22] and where the airway remodelling in asthma is most damaging.

Acute exposure of bronchial epithelial cells from patients with asthma to cigarette smoke in vitro reduces the MMP-9/TIMP-1 ratio [16]. Based on this finding, it was postulated that a reduction in the MMP-9/TIMP ratio may contribute to remodelling in smokers with asthma due to increased deposition of ECM and airway wall thickness [16]. However, our findings did not support this hypothesis, in that we could not demonstrate any association between MMP-9/TIMP ratio and increased CT airway wall thickening in smokers with asthma. Instead, an important finding in the smokers with asthma group were the positive correlations between sputum MMP-9 activity/TIMP-1 and TIMP-2, MMP-9 concentration/TIMP-1 and TIMP-2 ratios, and CT measurements of segmental airway lumen area. The link between reduced MMP-9/ TIMP ratios and a reduced airway lumen in smokers with asthma is not explained by our data. Lower sputum MMP-9 activity/TIMP-1 and TIMP-2 ratios were associated with reduced FEV1 \% pred in smokers with asthma, an association reported by others in nonsmokers with asthma $[13,14,23]$. In addition, we showed that lower ratios were associated with reduced post-bronchodilator FEV1/FVC, implying persistent airflow obstruction in subjects with lower ratios. Possibly a low MMP-9/TIMP ratio is associated with the bronchoconstrictor effects of cigarette smoke, increased secretions or epithelial inflammation, although the latter might also be expected to increase airway wall thickness. Body surface area could influence airway dimensions, but we found no association with airway lumen area. The excess of TIMP over MMP-9 may be an exaggerated attempt to protect the bronchi against metalloproteinase degrading activity, leading to increased extracellular matrix deposition [12].

Unlike the results obtained by Vignola et al. [8] in mild, steroid naïve asthmatics, we did not observe a difference in asthmatic nonsmokers compared with healthy controls, possibly as the laboratory techniques were different and majority of our subjects were on inhaled corticosteroids. Inhaled corticosteroids have been shown to reduce the expression of MMP-9 and increase the expression of TIMP-1 in bronchial biopsy tissue from patients with asthma [24]. In the present study, the dose of inhaled corticosteroid did not have a significant effect on MMP-9 or TIMP measurements in sputum.

We found that the proportion of sputum neutrophils correlated positively with sputum MMP-9 activity/ TIMP-1 and MMP-9 concentration/TIMP-1 ratios in never-smokers with asthma, but not in smokers with 


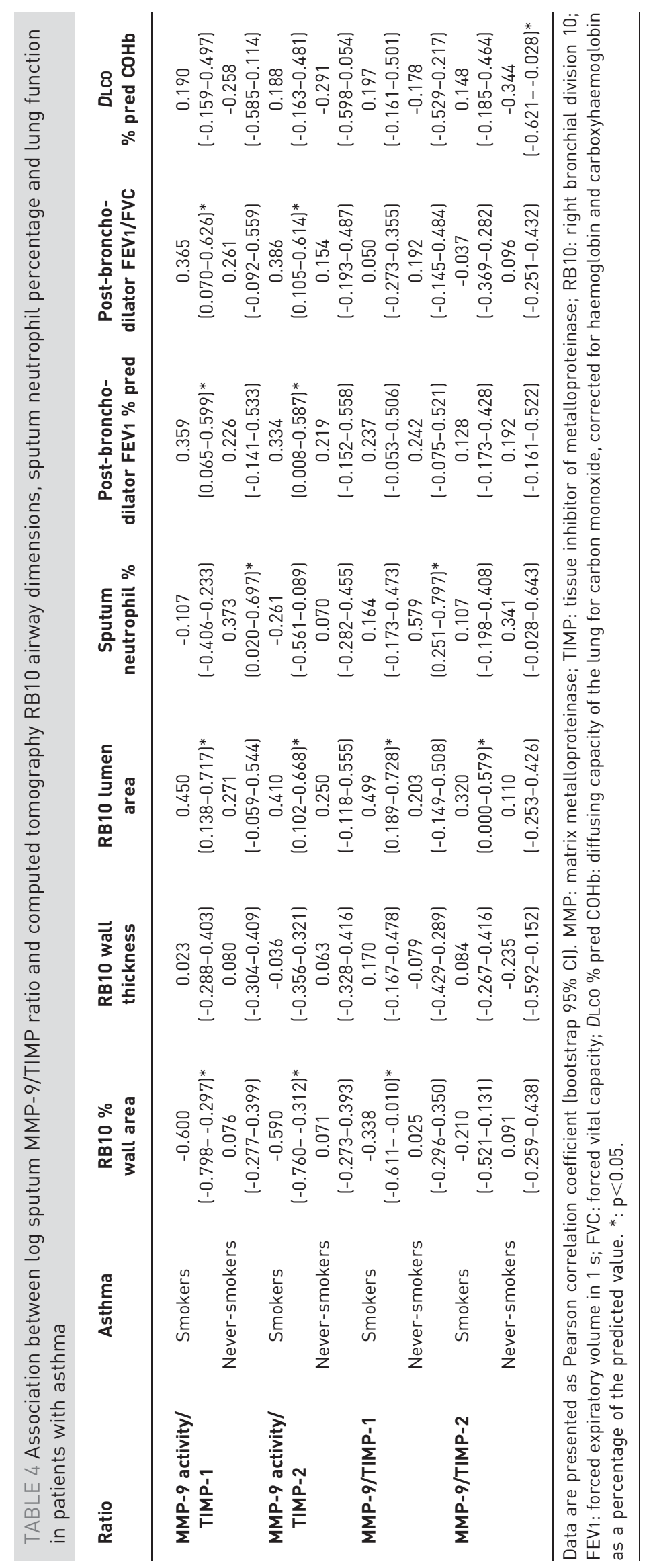



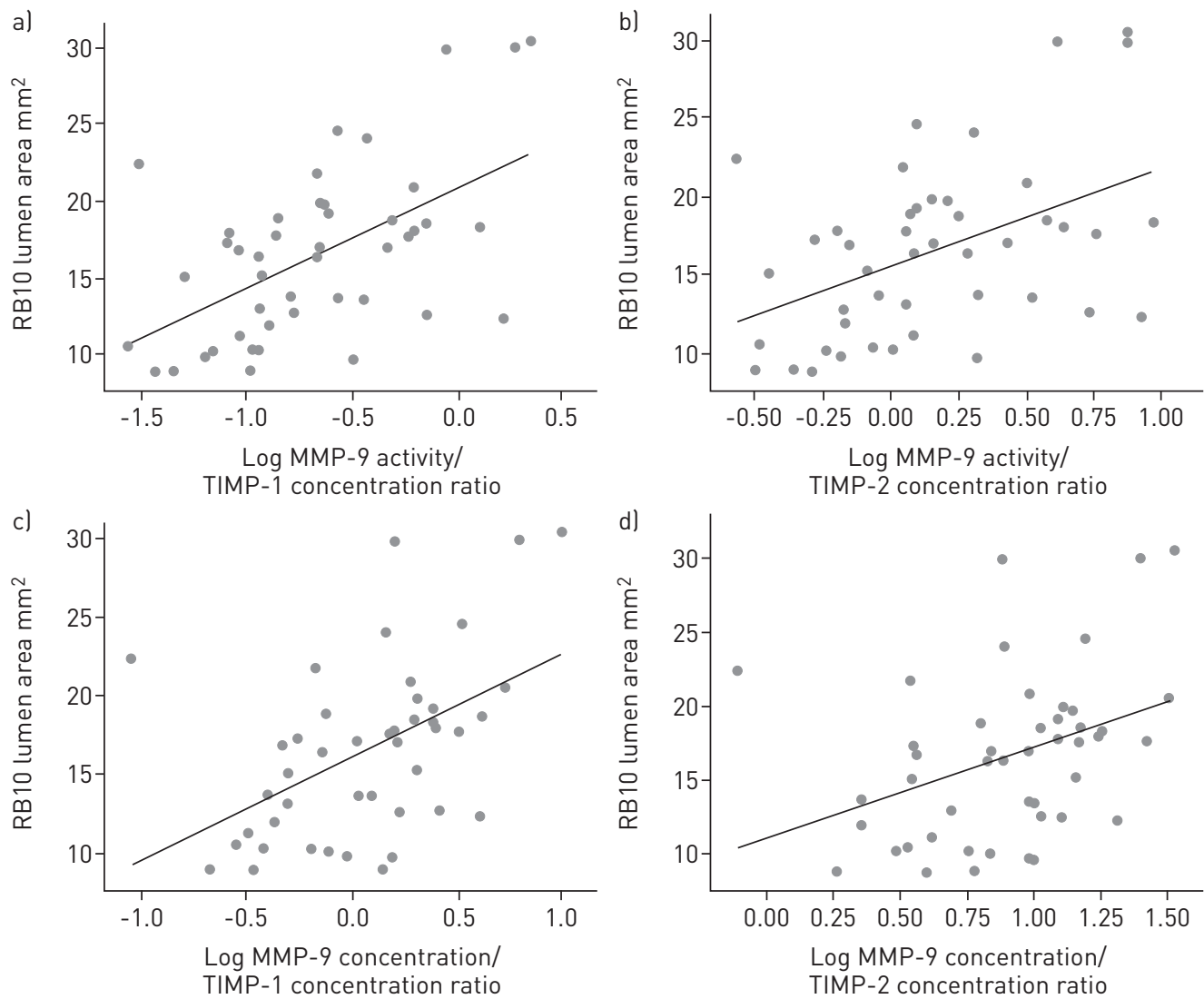

FIGURE 2 Association between a) sputum matrix metalloproteinase (MMP)-9 activity/tissue inhibitors of metalloproteinase (TIMP)-1 concentration ratio, b) MMP-9 activity/TIMP-2 concentration ratio, c) MMP-9 protein concentration/TIMP-1 concentration ratio, and d) MMP-9 protein concentration/TIMP-2 concentration ratio, with computed tomography (CT) airway lumen area in smokers with asthma. The ratios of sputum MMP-9 activity/TIMP-1 concentration, MMP-9 activity/TIMP-2 concentration, sputum MMP-9 protein concentration/TIMP-1 concentration, and MMP-9 protein concentration/TIMP-2 concentration correlated with right bronchial division 10 (RB10) airway lumen area in smokers with asthma $(\mathrm{r}=0.450(95 \%$ CI $0.138-0.717), \mathrm{r}=0.410(0.102-0.668), \mathrm{r}=0.499(0.189-0.728)$ and $\mathrm{r}=0.320(0.000-0.579)$, respectively).

asthma. Previous studies using sputum and bronchoalveolar lavage samples have reported a similar association in nonsmokers with asthma $[13,14,25]$. MMP-9 and TIMP-1 are known to be secreted by a variety of cells, including bronchial epithelial cells, eosinophils, mast cells and alveolar macrophages, and these may contribute more, relative to neutrophils, in orchestrating the changes in MMP-9 and TIMP-1 levels in smokers with asthma.

We recognise that there were limitations in the study, such as the fact that we did not perform bronchial biopsies to confirm the sputum, nasal and CT findings, and that the cross-sectional study design precludes collection of data on longitudinal changes to the balance between sputum MMP-9 and TIMPs. The inclusion of different severities of asthma reduces the numbers for conclusive statistical sub-analysis; however, it provides useful pilot data for larger studies of the effects of asthma severity.

In conclusion, these results show, for the first time, that the balance of MMP-9 and TIMPs is lower in smokers with asthma compared with never-smokers with asthma. A reduced sputum MMP-9 activity/TIMP ratio is associated with worse post-bronchodilator FEV1 and FEV1/FVC ratio and decreased sub-segmental CT measurements of airway lumen in smokers with asthma. This result suggests that an imbalance of MMP-9 and TIMPs may contribute to structural changes to the airways in this group.

\section{Acknowledgements}

The authors would like to thank Joyce Thompson, Jane Lafferty, Maureen Brannigan (University of Glasgow, Glasgow, UK) and Kathleen Masterson (NHS Greater Glasgow and Clyde) for sample collection, and Lisa Jolly and Iona Donnelly (University of Glasgow) for processing the samples and sputum cytology. 


\section{References}

1 Dockrell M, Partridge MR, Valovirta E. The limitations of severe asthma: the results of a European survey. Allergy 2007; 62: 134-141.

2 Chaudhuri R, McSharry C, McCoard A, et al. Role of symptoms and lung function in determining asthma control in smokers with asthma. Allergy 2008; 63: 132-135.

Barnes PJ, Adcock IM. Glucocorticoid resistance in inflammatory diseases. Lancet 2009; 73: 1905-1917.

Wenzel SE. Asthma phenotypes: the evolution from clinical to molecular approaches. Nat Med 2012; 18: 716-725.

Thomson NC, Spears M. The role of cigarette smoking on persistent airflow obstruction in asthma. Annals Respir Med 2011; 2: 47-54

6 Demedts IK, Brusselle GG, Bracke KR, et al. Matrix metalloproteinases in asthma and COPD. Curr Opin Pharmacol 2005; 5: 257-263

7 Gueders MM, Foidart J-M, Noel A, et al. Matrix metalloproteinases (MMPs) and tissue inhibitors of MMPs in the respiratory tract: potential implications in asthma and other lung diseases. Eur J Pharmacol 2006; 533: 133-144.

8 Vignola AM, Riccobono L, Mirabella A, et al. Sputum metalloproteinase-9/tissue inhibitor of metalloproteinase-1 ratio correlates with airflow obstruction in asthma and chronic bronchitis. Am J Respir Crit Care Med 1998; 158: $1945-1950$.

9 Mattos W, Lim S, Russell R, et al. Matrix metalloproteinase-9 expression in asthma: effect of asthma severity, allergen challenge, and inhaled corticosteroids. Chest 2002; 122: 1543-1552.

10 Atkinson JJ, Senior RM. Matrix metalloproteinase-9 in lung remodeling. Am J Respir Cell Mol Biol 2003; 28: 12-24.

11 Parameswaran K, Willems-Widyastuti A, Alagappan VK, et al. Role of extracellular matrix and its regulators in human airway smooth muscle biology. Cell Biochem Biophys 2006; 44: 139-146.

12 Chiappara G, Gagliardo R, Siena A, et al. Airway remodelling in the pathogenesis of asthma. Curr Opin Allergy Clin Immunol 2001; 1: 85-93.

13 Vignola AM, Riccobono L, Mirabella A, et al. Sputum metalloproteinase-9/tissue inhibitor of metalloproteinase-1 ratio correlates with airflow obstruction in asthma and chronic bronchitis. Am J Respir Crit Care Med 1998; 158 : $1945-1950$

14 Matsumoto H, Niimi A, Takemura M, et al. Relationship of airway wall thickening to an imbalance between matrix metalloproteinase-9 and its inhibitor in asthma. Thorax 2005; 60: 277-281.

15 Vignola AM, Paganin F, Capieu L, et al. Airway remodelling assessed by sputum and high-resolution computed tomography in asthma and COPD. Eur Respir J 2004; 24: 910-917.

16 Watson AM, Benton AS, Rose MC, et al. Cigarette smoke alters tissue inhibitor of metalloproteinase 1 and matrix metalloproteinase 9 levels in the basolateral secretions of human asthmatic bronchial epithelium in vitro. J Investig Med 2010; 58: 725-729.

17 Global Initiative for Asthma (GINA). Global Strategy for Asthma Management and Prevention. GINA, 2005. www. ginasthma.com.

18 Chaudhuri R, McSharry C, Brady J, et al. Sputum matrix metalloproteinase-12 in patients with chronic obstructive pulmonary disease and asthma: relationship to disease severity. J Allergy Clin Immunol 2012; 129: 655-663.

19 Miller MR, Hankinson J, Brusasco V, et al. Standardisation of spirometry. Eur Respir J 2005; 26: 319-338.

20 American Thoracic Society, European Respiratory Society. ATS/ERS recommendations for standardized procedures for the online and offline measurement of exhaled lower respiratory nitric oxide and nasal nitric oxide. Am J Respir Crit Care Med 2005; 171: 912-930.

21 Tanaka H, Miyazaki N, Oashi K, et al. Sputum matrix metalloproteinase-9: tissue inhibitor of metalloproteinase-1 ratio in acute asthma. J Allergy Clin Immunol 2000; 105: 900-905.

22 Wenzel SE, Balzar S, Cundall M, et al. Subepithelial basement membrane immunoreactivity for matrix metalloproteinase 9: association with asthma severity, neutrophilic inflammation, and wound repair. J Allergy Clin Immunol 2003; 111: 1345-1352.

23 Avilés B, Belda J, Margarit G, et al. Marcadores de remodelado bronquial en el esputo inducido de fumadores sanos [Markers of airway remodeling in induced sputum from healthy smokers]. Arch Bronconeumol 2006; 42: 235-240.

24 Hoshino M, Takahashi M, Takai Y, et al. Inhaled corticosteroids decrease subepithelial collagen deposition by modulation of the balance between matrix metalloproteinase- 9 and tissue inhibitor of metalloproteinase-1 expression in asthma. J Allergy Clin Immunol 1999; 104: 356-363.

25 Cataldo D, Bettiol J, Noël A, et al. Matrix metalloproteinase-9, but not tissue inhibitor of matrix metalloproteinase-1, increases in the sputum from allergic asthmatic patients after allergen challenge. Chest 2002; 122: 1553-1559. 